\title{
Aborder le raisonnement clinique du point de vue pédagogique*
}

\section{Un cadre conceptuel pour identifier les problèmes de raisonnement clinique}

\section{Tackling clinical reasoning from a pedagogical perspective \\ I. A conceptual framework to identify clinical reasoning problems}

\section{Marie-Claude AUDÉTAT, Suzanne LAURIN et Gilbert SANCHE}

Département de médecine familiale et de médecine d'urgence, CPASS (Centre de pédagogie appliquée aux sciences de la santé), Université de Montréal, Canada

Manuscrit reçu le 6 juillet 2011 ; commentaires éditoriaux formulés aux auteurs le 30 août et le 7 novembre 2011 ; accepté pour publication le 14 novembre 2011

\section{Mots-clés \\ Raisonnement \\ clinique ; diagnostic \\ pédagogique ; \\ supervision clinique \\ Keywords \\ Clinical reasoning; \\ educational \\ diagnosis; \\ clinical supervision}

Messages clés - Les cliniciens enseignants ont du mal à identifier les difficultés de raisonnement clinique des apprenants et, conséquemment, à élaborer des stratégies de remédiation. La prise de conscience de la ressemblance entre la démarche de raisonnement clinique et celle de raisonnement pédagogique facilite l'engagement dans un processus de détection de ces difficultés et la formulation de diagnostics pédagogiques plus précis qui conduiront à des plans pédagogiques ciblés.

\footnotetext{
Key messages - Clinical teachers struggle to pinpoint the clinical reasoning difficulties of learners and as a result develop remediation strategies.

Being aware of the similarity between the processes of clinical reasoning and educational reasoning helps clinical teachers go forward in detecting these problems and making accurate educational diagnoses and more targeted educational plans.
}

Cet article est le premier d'une série didactique de six chroniques. Ces chroniques s'appuient sur des travaux de recherche qui ont permis d'élaborer une taxonomie des difficultés de raisonnement clinique, de décrire un processus de diagnostic pédagogique et de distinguer des stratégies de remédiation spécifiques $^{[1]}$. Un outil d'aide au diagnostic pédagogique et au développement de stratégies de remédiation a été élaboré et est disponible sous forme de matériel éditorial complémentaire en ligne, annexé à la version électronique de l'article.

Ce premier article aborde le cadre conceptuel du diagnostic pédagogique des difficultés de raisonnement clinique, telles qu'elles se manifestent

* Annexe disponible en ligne sur la version électronique du présent article. 
quotidiennement dans le contexte d'apprentissage en milieu clinique.

\section{Introduction}

Les difficultés de raisonnement clinique affectent entre 5 et $15 \%$ des étudiants des facultés de médecine $^{[2]}$. Ces difficultés sont souvent identifiées tardivement dans le parcours de formation ${ }^{[3-5]}$ et c'est un véritable enjeu pour les enseignants que d'aller au-delà d'une perception globale d'un dysfonctionnement et de formuler le problème de façon spécifique. Comme le raisonnement clinique est au cœur de la pratique médicale, il apparaît particulièrement important de mener une réflexion pédagogique sur ce sujet ${ }^{[6-8]}$.

En cohérence avec leur rôle de clinicien, les superviseurs se sentent dans une zone de confort en ce qui concerne leur expertise de contenu et ceci explique vraisemblablement pourquoi ils ont tendance à centrer leurs supervisions sur ce contenu spécifique, lié à la résolution du problème médical du patient. En revanche, tel que l'illustre la figure 1, ces mêmes cliniciens-enseignants sont fréquemment dans une désagréable zone d'inconfort au moment de documenter les performances pédagogiques des étudiants, notamment en raison de méconnaissances concernant les modèles théoriques qui rendent compte des processus de raisonnement clinique et de diagnostic pédagogique, d'un manque d'outils, d'un doute quant à la validité de leur appréciation et finalement, d'incertitudes quant aux moyens de remédiation spécifiques à proposer ${ }^{[9,10]}$.

Des recherches récentes suggèrent que le sentiment de confiance des cliniciens-enseignants dans leurs capacités pédagogiques pourrait être un facteur déterminant de leur engagement dans des tâches pédagogiques $^{[11,12]}$.

Dans cette perspective, et pour aider les cliniciens enseignants, très à l'aise quand il s'agit de démarche clinique, à bien comprendre ce que l'on entend par démarche de raisonnement pédagogique, nous aborderons les similitudes des deux démarches respectives de raisonnement clinique et pédagogique qui vont mener à un diagnostic et à un plan d'intervention.

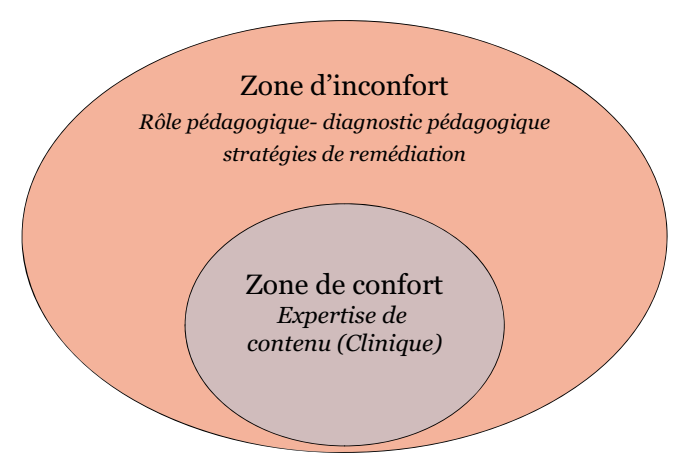

Fig. 1. Supervision du raisonnement clinique : les zones d'inconfort des cliniciens enseignants

Nous sommes convaincus que la prise de conscience de la ressemblance de ces deux démarches facilite le transfert des compétences réflexives d'un rôle à l'autre et favorise ainsi le développement d'un sentiment de confiance en soi et de maîtrise dans l'accomplissement des tâches d'enseignement.

\section{Comment poser un diagnostic pédagogique?}

La similarité des deux démarches de raisonnement clinique et pédagogique

La reconnaissance précoce des difficultés de raisonnement clinique d'un apprenant peut permettre l'établissement d'un plan pédagogique individualisé assez tôt dans la formation, de façon à soutenir sa progression vers le degré de maîtrise des compétences attendu pour son niveau de formation.

Inspiré du modèle hypothético-déductif, le modèle de raisonnement pédagogique proposé prévoit un recueil de données sur la performance de l'apprenant. Ce recueil des données peut s'exercer à l'occasion de différentes activités: supervision directe, discussion de cas clinique en supervision indirecte ou dans le cadre d'un groupe de pairs, etc. Lorsqu'il dépiste des difficultés, le clinicien-enseignant développe un raisonnement pédagogique pour chercher à comprendre la nature du problème de raisonnement en cause chez l'apprenant en difficulté. À l'instar de ce qui est développé lors du processus de raisonnement clinique, il s'appuie sur des indices 
Tableau I. Les étapes de la démarche diagnostique pédagogique.

\begin{tabular}{ll}
\hline Étapes & Démarche \\
\hline 1 & $\begin{array}{l}\text { Intuition-suspicion/détection de la difficulté de raisonnement } \\
\text { Recueil des données sur la difficulté de raisonnement (par génération et vérification d'hypothèses }\end{array}$ \\
\hline 3 & Impression diagnostique pédagogique \\
\hline 4 & Investigation/recherche d'explications et de causes \\
\hline 6 & Diagnostic final/représentation globale de la situation pédagogique \\
\hline
\end{tabular}

qu'il interprète pour émettre des hypothèses d'explication et les vérifie à l'aide d'un recueil orienté d'informations complémentaires pour arriver à un diagnostic pédagogique le plus rigoureux possible.

Toutefois, alors même que nous venons d'en souligner les analogies, la démarche de raisonnement pédagogique se distingue de celle du raisonnement clinique par le fait qu'elle est initiée par une étape cruciale et complexe, en l'occurrence la détection d'un problème ou d'une difficulté dont l'apprenant n'a pas toujours conscience et dont il ne se plaint dès lors pas, alors que la démarche de raisonnement clinique succède généralement à une plainte d'un patient. Cette phase d'alerte, de perception intuitive que quelque chose ne va pas, peut expliquer le délai souvent observé pour que soit posé un diagnostic pédagogique: de fait, pour devenir source de questionnement de la part du superviseur, une difficulté doit se manifester à plusieurs occasions et dans des contextes différents ou encore être observée par différents superviseurs qui partageront leurs constatations. Le tableau I résume les étapes de la démarche pédagogique.

Une fois qu'ils ont soupçonné une difficulté, les cliniciens-enseignants doivent apprendre à décoder et interpréter le discours, les comportements et les écrits de l'apprenant, qui sont autant de manifestations de difficultés de raisonnement clinique, pour arriver à préciser efficacement en quoi consiste le problème. Ainsi, par exemple, une multitude de questions posées sans ordre précis à un patient, un nombre restreint d'hypothèses diagnostiques ou encore une interprétation erronée des données recueillies en consultation peuvent être autant de manifestations d'un problème de raisonnement clinique dont il faut comprendre la cause avant de proposer une stratégie de remédiation.

Pour poser le bon diagnostic pédagogique, le clinicien-enseignant doit d'abord comprendre l'origine et le sens de ces manifestations. Pour ce faire, il doit faire un recueil des données, par des supervisions répétées, par des discussions avec ses collègues, pour mieux comprendre, par exemple, ce qui se cache sous une anamnèse longue, non orientée et apparemment sans but, que fait l'apprenant. Il pourrait selon les cas s'agir d'un manque de connaissances qui l'empêche de générer des hypothèses et le pousse à « aller à la pêche », ou encore d'une difficulté à appliquer le modèle de raisonnement hypothético-déductif lors de son recueil d'informations, ayant pour conséquences une anamnèse exhaustive suivie d'un laborieux exercice de synthèse a posteriori et de recherche d'un diagnostic qui intégrerait toutes ces données.

Diverses interventions sont possibles en supervision pour vérifier ces hypothèses pédagogiques. Le partage des observations entre collègues et les supervisions répétées, de même que les discussions de cas avec l'apprenant sont très utiles pour arriver à préciser la difficulté et sa source et à formuler une représentation globale du problème pédagogique. Un plan d'intervention spécifique pourra alors plus facilement être élaboré et proposé à l'apprenant.

La figure 2 illustre le parallèle entre les deux démarches que nous venons de décrire. 


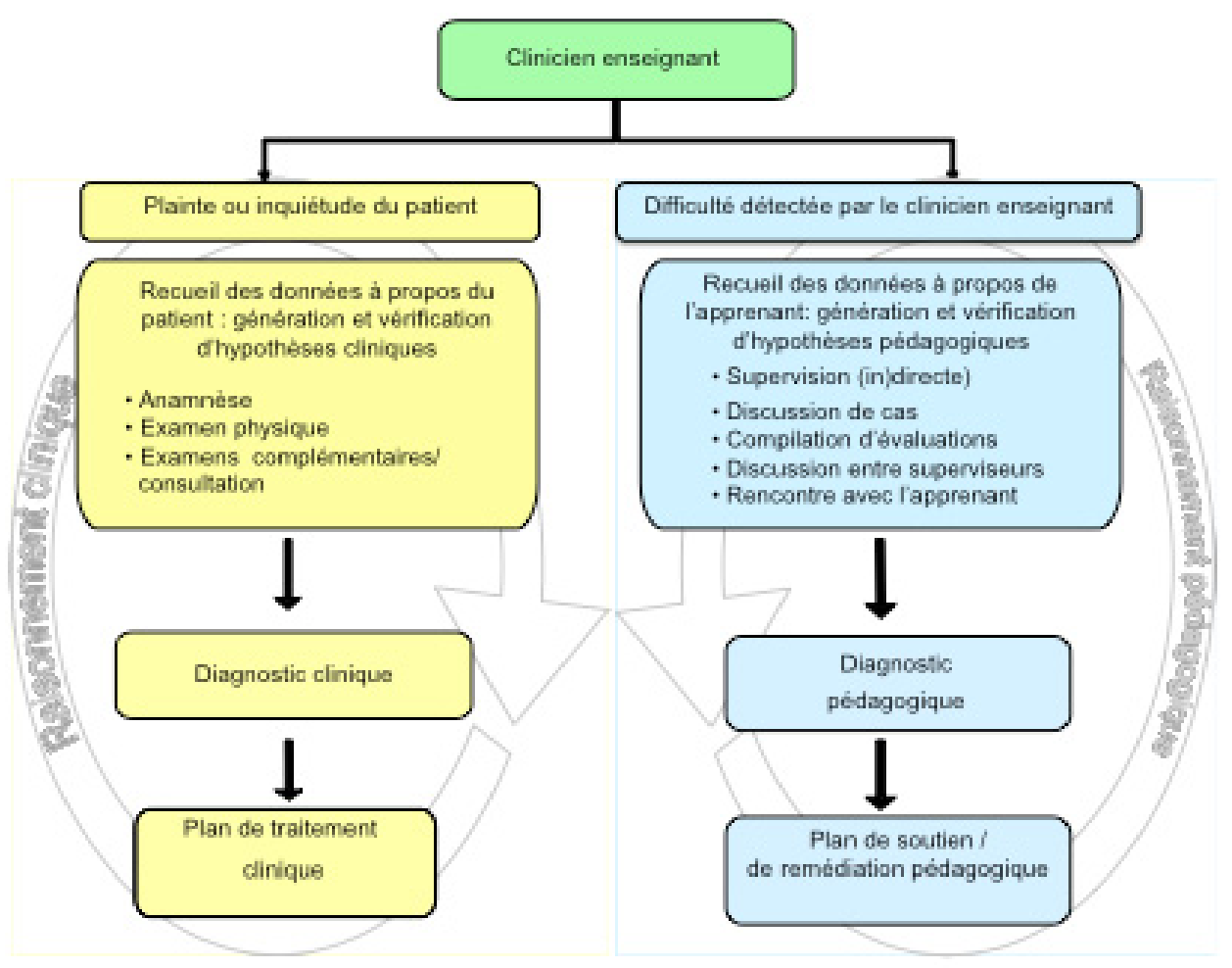

Fig. 2. Parallèle entre les étapes de raisonnement clinique et pédagogique.

\section{Une typologie-opérationnelle des difficultés de raisonnement clinique}

Au cours de sa formation, l'apprenant affine son raisonnement clinique et développe les différentes compétences (communication, expertise médicale, éthique, etc.) qui l'aideront à faire évoluer sa démarche. Des failles dans le raisonnement clinique peuvent donc faire partie de la progression normale d'un apprenant en cours de formation mais leur identification rapide peut permettre aux superviseurs de soutenir l'évolution du raisonnement vers le niveau de performance attendu, en évitant ainsi que des difficultés éventuelles ne se cristallisent et se transforment en impasses pédagogiques.

Dès lors, nous souhaitons proposer une démarche pour faciliter le processus d'identification précoce des difficultés de raisonnement clinique rencontrées et de formulation du diagnostic pédagogique lié aux problèmes.
Pour aider à structurer cette démarche de diagnostic pédagogique, nous proposons une typologie qui rend compte des difficultés de raisonnement clinique en fonction de l'étape du raisonnement durant laquelle les problèmes se manifestent (tableau II). Nous avons fait le choix de présenter la démarche pédagogique à effectuer à partir des problèmes sousjacents plutôt que des symptômes (par exemple, fermeture prématurée plutôt que diagnostic différentiel insuffisant), afin d'aider les cliniciens enseignants à développer un répertoire de difficultés de raisonnement clinique.

Dans cette perspective, en nous appuyant sur les travaux de Bordage qui recommande de s'attacher spécifiquement à ce qui a du sens dans le contexte de l'enseignement clinique pour la définition de catégories de difficultés ${ }^{[13]}$, nous avons distingué trois étapes dans le processus de raisonnement clinique, en leur faisant correspondre les difficultés les plus souvent observées lors des supervisions. Notons que ces 
Tableau II. Les étapes principales du raisonnement clinique et leurs potentielles difficultés.

Étapes Difficultés potentielles

Génération d'hypothèses et récolte de données

Raffinement et traitement des hypothèses

Diagnostic final et élaboration d'un plan d'intervention
1. Difficultés dans la perception des indices clés, dans la génération des hypothèses, et dans l'orientation de la récolte de données.

2. Problème de fermeture prématurée.

3. Difficulté de priorisation.

4. Difficulté à élaborer un portrait global de la situation clinique.

5. Difficulté à élaborer un plan d'intervention.

Tableau III. Éléments influant sur le raisonnement clinique.

\begin{tabular}{llll}
\hline Connaissances & Facteurs internes & $\begin{array}{l}\text { Habiletés/ } \\
\text { compétences/contexte }\end{array}$ & Facteurs interrelationnels \\
\hline $\begin{array}{l}\text { Connaissances } \\
\text { insuffisantes }\end{array}$ & Problèmes d'attitude & $\begin{array}{l}\text { Gestion de l'entrevue } \\
\text { avec le patient } \\
\begin{array}{l}\text { Organisation déficiente } \\
\text { des connaissances }\end{array}\end{array}$ & $\begin{array}{l}\text { Dynamique relationnelle patient- } \\
\text { apprenant }\end{array}$ \\
$\begin{array}{l}\text { Méconnaissance du communication } \\
\text { processus de } \\
\text { raisonnement clinique }\end{array}$ & $\begin{array}{l}\text { Inexpérience de } \\
\text { l'apprenant }\end{array}$ & $\begin{array}{l}\text { Dynamique relationnelle } \\
\text { enseignant-apprenant }\end{array}$ \\
\hline
\end{tabular}

catégories ne sont pas mutuellement exclusives et qu'elles peuvent se chevaucher.

Dans la perspective d'une meilleure compréhension de ces difficultés, il semble pertinent de se demander comment elles se manifestent dans le déroulement du raisonnement clinique, d'identifier les indices ou les manifestations qui permettent de les distinguer et d'en inférer les causes.

La littérature relève différentes catégories d'éléments qui influent sur le raisonnement clinique ${ }^{[14-17]}$. Le tableau III en présente quelques-unes.

Ainsi, on identifie respectivement :

1) Des difficultés liées aux connaissances. Les connaissances sont indispensables à la poursuite du raisonnement mais il est déterminant de distinguer le déficit purement « quantitatif » de connaissances, moins fréquent que l'on ne le croit, d'un problème lié à l'organisation de ces connaissances ou d'une difficulté à intégrer, activer ou intégrer ces connaissances dans un processus d'analyse de la situation clinique.

2) Des difficultés liées à des problèmes d'attitude. Une confiance excessive, une insécurité ou un malaise à aborder certaines questions en consultation, peuvent perturber le raisonnement ${ }^{[18]}$.

3) Des difficultés liées aux biais cognitifs. On entend par biais cognitifs les facteurs qui viennent nuire à la souplesse du raisonnement et qui, par exemple, pourraient amener l'apprenant à s'accrocher à une hypothèse diagnostique en négligeant certains indices qui devraient la remettre en question, à donner trop ou pas assez d'importance à certains indices cliniques ou encore à reconnaître dans des situations différentes une pathologie apprise ou rencontrée récemment ${ }^{[19]}$.

4) Des facteurs liés à l'inexpérience de l'apprenant. Le jeune clinicien peut, par exemple, poser les questions dans un ordre établi, rigide, pour se 
sécuriser, pour éviter de perdre le fil, rendant difficile le traitement des hypothèses au moment où elles devraient se présenter à son esprit.

5) Des facteurs liés à la méconnaissance des processus de raisonnement clinique. Certains apprenants ne savent pas utiliser le modèle hypothéticodéductif en clinique. Nous observons souvent cette manière de faire chez les externes qui procèdent à un questionnaire détaillé, voulant faire la somme des éléments recueillis, une fois l'anamnèse terminée, pour proposer a posteriori une hypothèse diagnostique plutôt que de rechercher, au fur et à mesure, les éléments qui leur permettraient d'entretenir ou d'exclure une hypothèse.

6) Des facteurs liés à la gestion de l'entrevue avec le patient. Le clinicien doit pouvoir obtenir de son patient les renseignements qui lui permettront de faire avancer son raisonnement. Une difficulté à gérer le processus, par exemple lorsque l'on se « perd » dans les multiples demandes ou plaintes d'un patient, peut nuire au bon déroulement du raisonnement clinique.

7) Des facteurs liés à des problèmes de communication. Des stratégies de communication efficaces sont nécessaires à un raisonnement clinique performant ${ }^{[20]}$. Ne pas savoir comment établir une relation de confiance avec le patient de façon à obtenir des informations déterminantes peut, par exemple, avoir un impact sur le raisonnement.

8) Des facteurs liés à l'apprenant, au patient et à leur dynamique relationnelle. Un apprenant intimidé par un patient autoritaire pourrait soutenir une hypothèse peu probable par excès de prudence. Un apprenant surmené, et donc moins concentré, risque de minimiser ou de s'inquiéter davantage des problèmes de son patient.

9) Des facteurs liés à la dynamique relationnelle entre l'apprenant et l'enseignant dans le cadre de la supervision. Une anxiété de performance chez un apprenant qui se sait supervisé peut avoir pour effet de paralyser le raisonnement ; des attentes irréalistes de la part de l'enseignant peuvent nuire au processus décisionnel d'un apprenant.

Ces distinctions sur la variété des mécanismes impliqués dans les difficultés de raisonnement clinique enrichissent le raisonnement pédagogique, permettent une meilleure compréhension de la nature du problème à aborder avec l'apprenant et, par conséquent, facilitent l'élaboration d'un plan de remédiation ciblé.

La littérature actuelle s'intéresse surtout à la compréhension et à la caractérisation du raisonnement clinique tel qu'il se développe dans l'esprit du médecin expert ou du médecin en formation. Dans le contexte de l'enseignement clinique, les enseignants n'ont pas un accès direct à ce qui se passe dans l'esprit de leurs stagiaires. Ils doivent pouvoir, à partir d'observations et de discussions avec les apprenants, identifier les manifestations de difficultés, les interpréter, les catégoriser et en comprendre l'origine pour pouvoir planifier des interventions pédagogiques spécifiques.

Dans cette série d'articles, nous aborderons spécifiquement plusieurs difficultés de raisonnement clinique telles qu'elles se présentent habituellement et nous chercherons à illustrer les indices permettant de mieux les identifier dans les différents contextes de supervision : après observation de l'apprenant en interaction avec son patient (supervision directe), à partir du compte rendu différé de l'entrevue formulé par l'apprenant (supervision indirecte) et à partir du dossier. Nous proposerons des pistes de réflexion pour mieux les comprendre et suggérerons des actions spécifiques pour y remédier.

Quelques techniques de supervision peuvent permettre de révéler et de soutenir le raisonnement clinique des apprenants ${ }^{[21,22]}$. Nous n'aborderons dans ces articles que celles qui s'intègrent dans une démarche d'identification des difficultés.

Nous espérons que ces chroniques aideront les cliniciens enseignants à se sentir plus à l'aise dans la démarche d'identification des éventuelles difficultés de raisonnement clinique de leurs apprenants lors des interventions de supervision au quotidien.

\section{Contributions}

Les trois auteurs ont conjointement mené des discussions sur le contenu et la structuration de l'article. Marie-Claude Audétat a rédigé une première version qui a fait l'objet d'une révision par Suzanne Laurin et Gilbert Sanche. La version finale du manuscrit a été approuvée par les trois auteurs. 


\section{Références}

1. Audétat MC, Laurin S, Sanche G, Béïque C, Caire-Fon $\mathrm{N}$, Blais J, et al. Clinical reasoning difficulties: a taxonomy for clinical teachers. Med Teach 2011: Soumis pour publication.

2. Szumacher E, Catton P, Jones G, Bradley R, Kwan J, Cherryman F, et al. Helping learners in difficultyThe incidence and effectiveness of remedial programmes of the medical radiation sciences programme at University of Toronto and the Michener institute for applied sciences, Toronto, Ontario, Canada. Ann Acad Med Singapore 2007;36:725-34.

3. Frellsen SL, Baker EA, Papp KK, Durning SJ. Medical school policies regarding struggling medical students during the internal medicine clerkships: results of a national survey. Acad Med 2008;83:876-81.

4. Hauer K, Teherani A, Kerr K, O'Sullivan P, Irby D. Student performance problems in medical school clinical skills assessments. Acad Med 2007;82:S69-S72.

5. Hicks PJ, Cox SM, Espey EL, Goepfert AR, Bienstock JL, Erickson SS, et al. To the point: medical education reviews-dealing with student difficulties in the clinical setting. Am J Obstet Gynecol 2005; 193:1915-22.

6. Croskerry PMDP. The importance of cognitive errors in diagnosis and strategies to minimize them. Acad Med 2003;78:775-80.

7. Scott I. Errors in clinical reasoning: causes and remedial strategies. BMJ 2009;339:22.

8. Graber M. Diagnostic errors in medicine: a case of neglect. Jt Comm J Qual Patient Saf 2005;31:106-13.

9. Dudek N, Marks M, Regehr G. Failure to fail: the perspectives of clinical supervisors. Acad Med 2005;80:S84-S7.

10. Audétat MC, Faguy A, Jacques A, Blais J, Charlin B. Étude exploratoire des perceptions et pratiques de médecins cliniciens enseignants engagés dans une démarche de diagnostic et de remédiation des lacunes du raisonnement clinique. Pédagogie Médicale 2011;12:7-16.

11. Stone S, Ellers B, Holmes D, Orgren R, Qualters D, Thompson J. Identifying oneself as a teacher; the perceptions of preceptors. Med Educ 2002;36:18085.

12. Vanek E, Snyder C, Hull A, Hekelman F. The relationship between teachers' confidence and use of clinical teaching skills in ambulatory care settings. Teach Learn Med 1996;8:137-41.

13. Bordage G. Why did I miss the diagnosis? Some cognitive explanations and educational implications. Acad Med 1999;74:S138-S43.

14. Norman G, Eva K. Diagnostic error and clinical reasoning. Med Educ 2010;44:94-100.

15. Graber M, Franklin N, Gordon R. Diagnostic error in internal medicine. Archives Int Med 2005; 165:1493-9.

16. Malterud K. Reflexivity and metapositions; strategies for appraisal of clinical evidence. J Eval Clin Pract 2002;8:121-6.

17. Kalf A, Spruijt-Metz D. Variation in diagnoses: influence of specialist's training on selection and ranking relevant information in geriatric case vignettes. Soc Sci Med 1996;42:705-12.

18. Berner E, Graber M. Overconfidence as a cause of diagnostic error in medicine. Am J Med 2008; 121(Suppl):2-33.

19. Kempainen R, Migeon M, Wolf F. Understanding our mistakes: a primer on errors in clinical reasoning. Med Teach 2003;25:177-81.

20. Windish D, Price E, Clever S, Magaziner J, Thomas P. Teaching medical students the important connection between communication and clinical reasoning. J Gen Intern Med 2005;20:1108-13.

21. Audétat MC, Laurin S. Supervision du raisonnement clinique : Méthodes et outil pour soutenir et stimuler un raisonnement clinique efficace. Le Médecin de famille canadien 2010;56:294-96.

22. Audétat MC, Laurin S. Clinicien et superviseur, même combat ! Le Médecin du Québec 2010;45:53-7.

Correspondance et offprints : Marie-Claude Audétat, Centre de pédagogie appliquée aux sciences de la santé (CPASS), Faculté de médecine, Université de Montréal. CP 6128, succ. Centre Ville, Montréal (QC) H3C 3J7, Canada. Mailto :

mcaudetat@sympatico.ca 